\title{
KiDS+VIKING-450: Improved cosmological parameter constraints from redshift calibration with self-organising maps
}

\author{
Angus H. Wright ${ }^{1}$, Hendrik Hildebrandt ${ }^{1}$, Jan Luca van den Busch ${ }^{1}$, Catherine Heymans ${ }^{1,2}$, Benjamin Joachimi ${ }^{3}$, \\ Arun Kannawadi ${ }^{4}$, and Konrad Kuijken ${ }^{5}$ \\ ${ }^{1}$ Ruhr-Universität Bochum, Astronomisches Institut, German Centre for Cosmological Lensing (GCCL), Universitätsstr. 150, 44801 \\ Bochum, Germany \\ e-mail: awright@astro.rub.de \\ 2 Institute for Astronomy, University of Edinburgh, Royal Observatory, Blackford Hill, Edinburgh EH9 3HJ, UK \\ 3 Department of Physics and Astronomy, University College London, Gower Street, London WC1E 6BT, UK \\ ${ }^{4}$ Department of Astrophysical Sciences, Peyton Hall, Princeton University, Princeton, NJ 08544, USA \\ 5 Leiden Observatory, Leiden University, Niels Bohrweg 2, 2333 CA Leiden, The Netherlands
}

Received 8 May 2020 / Accepted 22 July 2020

\begin{abstract}
We present updated cosmological constraints for the KiDS+VIKING-450 cosmic shear data set (KV450) estimated through redshift distributions and photometric samples defined using self-organising maps (SOMs). Our fiducial analysis finds marginal posterior constraints of $S_{8} \equiv \sigma_{8} \sqrt{\Omega_{\mathrm{m}} / 0.3}=0.716_{-0.038}^{+0.043}$, which are smaller but otherwise consistent with previous works that have applied this data set $\left(\left|\Delta S_{8}\right|=0.023\right)$. We analysed additional samples and redshift distributions set up in three ways: (1) by excluding certain spectroscopic surveys during redshift calibration; (2) by excluding lower-confidence spectroscopic redshifts in redshift calibration; and (3) by considering only those photometric sources which are jointly calibrated by at least three spectroscopic surveys. In all cases, the method utilised here has been proven to be robust: we find a maximal deviation from our fiducial analysis of $\left|\Delta S_{8}\right| \leq 0.011$ for all samples defined and analysed using our SOM. To demonstrate the reduction in systematic biases found within our analysis, we highlight our results when performing redshift calibration without the DEEP2 spectroscopic data set. In this case, we find marginal posterior constraints of $S_{8}=0.707_{-0.042}^{+0.046}$; this is a difference, with respect to the fiducial, that is both significantly smaller and in the opposite direction with regard to the equivalent shift from previous works. These results suggest that our improved cosmological parameter estimates are not sensitive to pathological misrepresentations of photometric sources by the spectroscopy used for direct redshift calibration and, therefore, that this systematic effect cannot be responsible for the observed difference between $S_{8}$ estimates made with KV450 and Planck CMB probes.
\end{abstract}

Key words. gravitational lensing: weak - cosmology: observations - surveys

\section{Introduction}

Estimations of cosmological parameters using tomographic cosmic shear require an accurate calibration of source redshift distributions. For Stage III cosmic shear surveys, such as the Kilo Degree Survey (KiDS; Kuijken et al. 2019), the Dark Energy Survey (DES; Flaugher et al. 2015), and the Hyper-Suprime Camera Wide-Survey (HSC; Aihara et al. 2018), coherent biases on the order of $\Delta\langle z\rangle=\langle z\rangle_{\text {est }}-\langle z\rangle_{\text {true }} \sim 0.04$ are enough to lead to significant shifts in estimated cosmological parameter estimates (see, e.g. Hildebrandt et al. 2017). Systematic shifts of this nature are important given the observed (currently mild) tension between cosmological parameters estimated using KiDS weak lensing and cosmic microwave background (CMB) studies (Planck Collaboration VI 2020). For this reason, considerable effort has been invested into developing, testing, and optimising redshift calibration methodologies for cosmic shear. These methods can typically be grouped into three categories, each based on: (1) cross-correlation (see e.g. Schneider et al. 2006; Newman 2008; McQuinn \& White 2013; Morrison et al 2017), (2) stacking individual redshift probability distributions (see e.g. Hildebrandt et al. 2012; Hoyle et al. 2018; Tanaka et al. 2018), or (3) direct calibration using spectroscopic redshift training samples (see e.g. Lima et al. 2008; Hildebrandt et al. 2017, 2020; Buchs et al. 2019; Wright et al. 2020). More recently, however, steps have been taken towards constructing a fourth, hybrid category which leverages both cross-correlation and direct calibration (Rau et al. 2019; Sánchez \& Bernstein 2018; Alarcon et al. 2019).

The methodological differences, and implicit assumptions, among these methods of estimation and calibration mean that each of them is susceptible to subtly different biases and systematic effects. For direct calibration methods, the completeness and pre-selection of the spectroscopic training sample has been of particular concern (see e.g. Gruen \& Brimioulle 2017; Hartley et al. 2020). In Wright et al. (2020), we developed an updated implementation of the direct calibration procedure utilising self-organising maps (SOMs; Kohonen 1982), which we found to be less susceptible to bias than previous implementations. We achieved this thanks to a direct flagging and removal of photometric sources which are not directly associated with a spectroscopic calibrator, thereby constructing a sample of fully 
represented photometric sources and an associated redshift distribution, that is, the 'gold' sample.

In this Letter, we apply the methodology of Wright et al. (2020) to the combined optical (from KiDS) and near-infrared (from VIKING, Edge et al. 2013) data set of Wright et al. (2019) and perform a tomographic cosmic shear analysis akin to that of Hildebrandt et al. (2020). The data set used here is described in Sect. 2, as is the definition of our various photometric and spectroscopic analysis samples. Our results are presented in Sect. 3, and we summarise the results presented in this Letter in Sect. 4.

\section{Data set and analysis methodology}

The KiDS+VIKING-450 data set (hereafter KV450) is presented in Wright et al. (2019), and (Hildebrandt et al. 2020, hereafter H20). We utilised the cosmic shear data products from H20 with lensfit shape measurements (Miller et al. 2007, 2013), spectroscopic training samples (Vanzella et al. 2008; Lilly et al. 2009; Popesso et al. 2009; Balestra et al. 2010; Le Fèvre et al. 2013; Newman et al. 2013; Kafle et al. 2018), and photometric redshifts from the BPZ code of Benítez (2000), as well as the core of the $\mathrm{H} 20$ parameter inference pipeline. We updated only the redshift distributions using the new direct redshift calibration methodology of Wright et al. (2020, hereafter W20). Our code was released as a stand-alone analysis package $^{1}$, with a wrapper pipeline ${ }^{2}$ to perform the analyses presented in this work. We provide the details of these cosmological analysis pipelines in Appendix A.

In this analysis, we utilised a range of spectroscopic data sets, compiled in different ways, to construct redshift distributions and photometric source 'gold samples' for the cosmic shear analysis. A full description of the methods used to construct these redshift distributions and gold samples is presented in W20. To summarise, we utilised self-organising maps (SOMs), trained on the colours and magnitudes of sources in the various spectroscopic data sets, to associate photometric galaxies to spectroscopic galaxies with known redshifts. The SOM associations are based on spectroscopic and photometric source assignments to specific SOM cells (galaxies falling within the same SOM cell are assigned to the same associations; see Appendix B of W20) and, therefore, linking photometric and spectroscopic sources with self-similar colour and magnitude properties. Using these associations, we were able to re-weight the spectroscopic redshift distribution to approximate the (unknown) photometric galaxy redshift distributions. Simultaneously, this allowed us to flag and remove photometric data which are not associated to spectra (and, therefore, which are not represented by the reweighted redshift distributions).

W20 demonstrated that their redshift calibration methodology is less susceptible to systematic biases in redshift distribution reconstruction when compared with previously incorporated methods used by KiDS (Hildebrandt et al. 2017;H20). We were able to further this analysis, also using the simulations of van den Busch et al. (2020) and estimate biases introduced by calibrating redshift distributions with different spectroscopic calibration samples. This allowed us to extend our cosmological analysis to include more informative priors on the redshift distribution bias parameters, rather than incorporating only the zero-mean priors used by H20. Details of these new non-zero-mean priors (' $\delta z \neq 0$ ') are given in Appendix B. We adopt the use of these improved priors for the majority of our

\footnotetext{
1 https://wWw.github. com/AngusWright/CosmoPipe

2 https://WwW.github.com/AngusWright/CosmoWrapper
}

analyses, where possible. In cases where we are unable to derive updated redshift priors (because of limitations in the simulations of van den Busch et al. 2020), we revert to using the broader, zero-mean priors (' $\delta z=0$ ') from $\mathrm{H} 20$.

Finally, the construction of our gold photometric source subsamples requires the simultaneous recalibration of both multiplicative and additive shear measurement bias parameters. While we were able to perform the additive shear bias measurement on-the-fly within our cosmology pipeline, computation of the multiplicative shear biases is more involved. We therefore precomputed the required multiplicative shear bias values, using the methodology and simulations of Kannawadi et al. (2019), for each of our photometric gold samples. These bias parameters are also given in Appendix B.

In this work, we perform cosmic shear parameter estimation using a number of different photometric gold samples, redshift distributions, and priors. Following the analysis of W20, our fiducial analysis defines the gold sample as those photometric data which are associated with one or more sources within the full KV450 spectroscopic compilation and whose spectroscopicto-photometric associations satisfy an empirically derived quality requirement. This requirement filters out associations whose mean photometric redshift $\left\langle Z_{\mathrm{B}}^{\mathrm{p}}\right\rangle_{i}$ (from template fitting with BPZ; Benítez 2000) catastrophically disagrees with the mean spectroscopic redshift of the association $\left\langle z_{\text {spec }}^{\mathrm{s}}\right\rangle_{i}$. Such a criteria was found in W20 to correlate most strongly (in their simulations) with a true bias of photometric-to-spectroscopic associations, outperforming (in particular) the measured and true spectroscopic redshift dispersions per association (which were found, counter-intuitively, to be uncorrelated with bias in the association mean redshift estimates). We therefore implement the quality requirement:

$\left|\left\langle z_{\text {spec }}^{\mathrm{s}}\right\rangle_{i}-\left\langle Z_{\mathrm{B}}^{\mathrm{p}}\right\rangle_{i}\right| \leq \max \left[5 \times \mathrm{nMAD}\left(\left\langle z_{\text {spec }}^{\mathrm{s}}\right\rangle-\left\langle Z_{\mathrm{B}}^{\mathrm{s}}\right\rangle\right), 0.4\right]$

for each of the $i \in[1, N]$ association sets, where $z_{\mathrm{spec}}^{\mathrm{s}}$ is the spectroscopic redshift of the spectroscopic sources, $Z_{\mathrm{B}}^{\mathrm{s}}$ is the photometric redshift of the spectroscopic sources, and $Z_{\mathrm{B}}^{\mathrm{p}}$ is the photometric redshift of the photometric sources. This requirement is the same as the one presented in W20, except that we have imposed a floor on the threshold which defines catastrophic failure; we take as our threshold the maximum of 0.4 and five times the $z_{\mathrm{spec}}^{\mathrm{s}}-Z_{\mathrm{B}}^{\mathrm{s}}$ dispersion (determined using the normalised median absolute deviation from median; $\mathrm{nMAD}^{3}$ ). Redshift distributions are then calculated per tomographic bin $\left(Z_{\mathrm{B}} \in(0.1,0.3],(0.3,0.5],(0.5,0.7],(0.7,0.9],(0.9,1.2]\right)$, as are the photometric gold samples.

In addition to our fiducial analysis, we explore three gold samples constructed from spectroscopic compilations excluding the zCOSMOS (Lilly et al. 2009), VVDS (Le Fèvre et al. 2013), and DEEP2 (Newman et al. 2013) data sets, respectively. We implement these samples both to compare them with similar samples run by $\mathrm{H} 20$ and to test the sensitivity of our results to pathologically under-representative spectroscopy. Next, we construct one gold sample ('specquality4') using only spectra with the highest quality flags from their various surveys (referred to as $\mathrm{nQ} \geqq 4$ spectra, which have $\geq 99.5 \%$ confidence) to test the sensitivity of our analysis to spectra with a slightly higher likelihood of catastrophic failures. Finally, we construct a highly restrictive gold sample ('multispec3'), which consists only of sources

\footnotetext{
$3 \sigma_{\text {nMAD }}=1.4826 \times \operatorname{med}(|x-\operatorname{med}(x)|)$. The pre-factor ensures normal consistency; that is $E\left[\operatorname{nMAD}\left(x_{1}, \ldots, x_{n}\right)\right]=\sigma$ for $X \sim N\left(\mu, \sigma^{2}\right)$ and large $n$.
} 

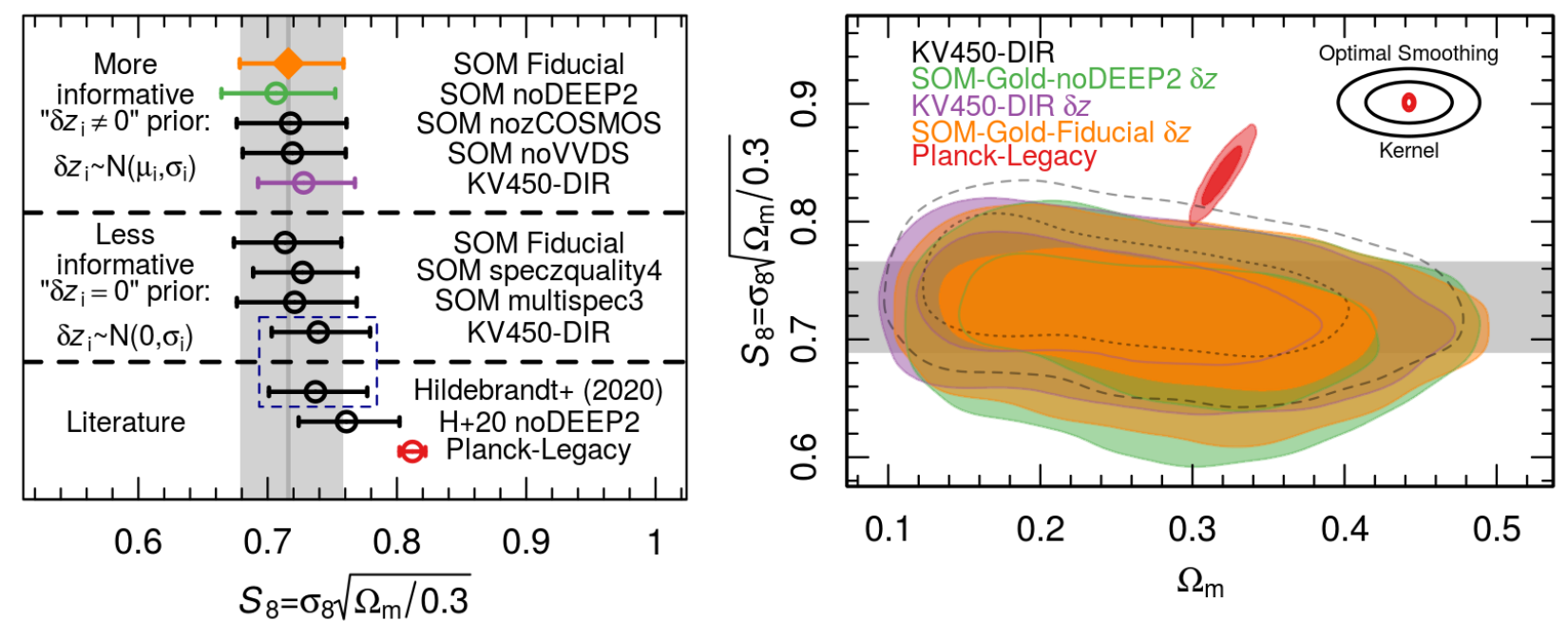

Fig. 1. Posterior constraints of $S_{8}$ (left) and $\Omega_{\mathrm{m}}$ vs. $S_{8}$ (right) for our various gold samples, compared to the results from H20 and Planck CMB. We show results for our analyses split into sections, determined by the form of their redshift distribution priors. Results computed using more informative, non-zero mean, Gaussian redshift distribution bias priors (' $\delta z \neq 0$ ', see Appendix B) for gold samples where these are able to be calculated, and using the zero mean Gaussian bias priors from $\mathrm{H} 20$ otherwise (' $\delta z=0$ '). For our fiducial analysis, we show results with both priors to allow for direct comparisons between our various results. We annotate our contour figure (right) with the two Gaussian smoothing kernels used in generating the contours (one for the cosmic shear contours and one for the CMB contours). We find that our new cosmology pipeline produces results that are consistent with the pipeline of H20 (left panel, blue dashed box). Our fiducial results (orange) suggest a slightly lower $S_{8}$ than found in previous works: $S_{8}=0.716_{-0.038}^{+0.043}$. However we find that constraints on $S_{8}$ are extremely stable for all of our gold sample analyses here (compared to the fiducial: $\left|\Delta S_{8}\right|<0.2 \sigma$ ), demonstrating that the results here are more robust with regard to spectroscopic misrepresentation than in previous works. In particular, unlike H20, we find that even pathological misrepresentation at high-redshift ('noDEEP2') is unable to shift our estimates of $S_{8}$ to larger values.

which reside in associations containing spectroscopy from (at least) three different spectroscopic surveys. This selection, coupled with our quality control requirement, essentially restricts our sample to sources whose calibration redshift is supported by multiple spectroscopic surveys with different selection functions, systematic effects, and catastrophic failure modes. This calibration sample is therefore expected to be very robust (albeit at some cost to statistical precision due to a significant reduction in photometric effective number density). A miscalibration of these data would require a coordinated catastrophic failure of redshift assignment across multiple spectroscopic campaigns using different instruments and redshifting methods.

\section{Results}

The results of our various gold sample cosmic shear measurements, quantified using the marginal posterior constraints of the cosmic-shear summary parameter of interest $S_{8}=\sigma_{8} \sqrt{\Omega_{\mathrm{m}} / 0.3}$, are shown in Fig. 1. Here, we also show the results from $\mathrm{H} 20$ and Planck-Legacy (Planck Collaboration VI 2020) for comparison. The left panel is split into three sections: (1) analyses performed with more informative non-zero mean redshift bias priors (' $\delta z \neq 0$ ', see Appendix B), estimated using the simulations of van den Busch et al. (2020); (2) analyses performed with the broader, zero-mean redshift bias priors of $\mathrm{H} 20$ (' $\delta z=0$ ', in cases where we cannot derive improved priors or when we wish to directly compare with previous works); (3) and external results taken directly from the literature.

First, we verify our updated cosmology pipeline by performing an identical cosmological analysis to H20. As seen by the two results highlighted by the blue box in Fig. 1, we find that we recover essentially the same $S_{8}$ as they report: $S_{8}=0.739_{-0.037}^{+0.040}$ (labelled 'KV450-DIR' in the figure, with $\delta z=0$ ) compared to their $S_{8}=0.737_{-0.036}^{+0.040}$ (Hildebrandt et al. 2020). We argue that the observed difference $\left(\left|\Delta S_{8}\right| \lesssim 0.003\right)$ is simply a reflection of noise within our Markov-chain Monte-Carlo (MCMC). For our fiducial gold sample analysis, shown in orange in both panels, we find a marginal constraint of $S_{8}=0.716_{-0.038}^{+0.043}$; this is smaller but otherwise consistent with what was found by H20. Our fiducial analysis is in better agreement with the results of $\mathrm{H} 20$ when their data set and redshift distributions are re-analysed with more informative redshift distribution bias priors (estimated from simulations, see Appendix B): $S_{8}=0.727_{-0.036}^{+0.039}$ ('KV450-DIR' with $\delta z \neq 0$; purple). We observe that our fiducial analysis has a slightly broader marginal $S_{8}$ constraint. This is to be expected when performing our gold selection: by decreasing the size of the photometric data set used for the analysis (which we quantify using the change in the effective number density of cosmic shear source galaxies, $\Delta n_{\mathrm{eff}}=n_{\mathrm{eff}}^{\text {gold }} / n_{\mathrm{eff}}^{\text {all }} \approx 80 \%$ for our fiducial sample; see Appendix C), we increase the statistical noise on our marginal constraints.

We explore the sensitivity of our analysis to the construction of our spectroscopic compilation by performing our analysis with gold samples constructed without spectra from zCOSMOS, VVDS, and DEEP2. When removing zCOSMOS or VVDS, we find that our marginal constraint on $S_{8}$ is unchanged within MCMC noise: $\left|\Delta S_{8}\right| \lesssim 0.003$. In the cases of removing DEEP2 from the calibration sample, we find a shift in our marginal constraint of $S_{8}=0.707_{-0.042}^{+0.046}$, equating to $\left|\Delta S_{8}\right| \lesssim 0.2 \sigma$. We note, however, that (looking at the $\Omega_{\mathrm{m}}$ versus $S_{8}$ plane) we can see that the shift in $S_{8}$ without DEEP2 is driven by an extension of the posterior to lower values, rather than a systematic biasing of the distribution overall. This result is of further interest in the context of the full multi-dimensional posterior space, which we present in Appendix D for the interested reader.

We draw particular attention to the differences seen between our analysis without DEEP2 and the equivalent analysis performed by $\mathrm{H} 20$. When performing their noDEEP2 analysis, 
$\mathrm{H} 20$ found a non-trivial increase in $S_{8}$ to $S_{8}=0.761_{-0.037}^{+0.041}$ ('H+20 noDEEP2'); a shift of $\Delta S_{8} \sim+0.6 \sigma$. This difference is attributed, in $\mathrm{H} 20$, to a bias in the reconstructed redshift distributions used for this test: removing DEEP2 causes pathological misrepresentation of the high-redshift portion of the spectroscopic colour-colour space, which subsequently causes the reconstructed redshift distributions to be systematically biased lower, thereby introducing a positive shift in $S_{8}$ for the otherwise unchanged photometric source sample. To this end, H20 argued that their noDEEP2 analysis was systematically biased and so, stood as a demonstration of the limitations of their redshift calibration procedure. In the SOM-based gold samples, however, we find no such bias in our results when calibrating without spectra from DEEP2. We attribute this difference to the gold selection process: our redshift distributions without DEEP2 are similarly skewed low compared to the fiducial (see Appendix C), however, unlike $\mathrm{H} 20$ our gold selection simultaneously removes the misrepresented photometric sources. Therefore, while our redshift distributions change significantly between the fiducial and noDEEP2 analyses, both correctly describe the photometric data within their respective gold samples; both are accurate and consistent. We therefore see no biasing of the derived cosmological parameters but, rather, merely an increase in marginal uncertainties due to the aforementioned decrease in statistical power attributed to the $\sim 20 \%$ reduction in the effective number density of the photometric sample.

In addition to testing for the effect of pathological colour misrepresentation, we also test the influence of spectra which may have an increased fraction of catastrophic failures. We note that in our spectroscopic compilation for KV450 we allowed only high-confidence $(\geq 95 \%)$ or 'certain' ( $\geq 99.5 \%$ confidence) spectra; however even high-confidence spectra may have catastrophic failures. In W20 we demonstrated through simulations that expected fractions of catastrophic spectroscopic failures were unlikely to bias calibration of redshift distributions in KV450. Nonetheless, here we explore the influence of the lower confidence spectroscopy on our conclusions. Our 'speczquality4' gold sample is calibrated using only certain confidence redshifts. The resulting marginal constraints of $S_{8}$ differ from our fiducial results by $\left|\Delta S_{8}\right|=0.011$. We therefore conclude that the presence of lower-confidence spectra in our calibration data set does not introduce significant biases in our fiducial marginal constraints of $S_{8}$.

Our speczquality4 result is of additional interest in the context of recent works presented by Hartley et al. (2020). For DES (i.e. using fewer photometric bands than used in KiDS) and implementing a redshift calibration methodology akin to that of $\mathrm{H} 20$, they find switching between direct calibration using highconfidence $(\geq 95 \%)$ and certain $(\geq 99.5 \%)$ spectroscopic samples results in a significant $\Delta\langle z\rangle \geq 0.06$ bias for their highest tomographic bin $\left(Z_{\mathrm{B}} \in(0.7,1.3]\right)$. While these biases are not directly applicable to our analysis, any similar systematic bias within our analysis would likely cause a significant change in the estimated cosmological parameters. We find no such significant bias when switching between direct calibration using high-confidence and certain spectroscopic redshifts, suggesting that this bias is suppressed in our data set. We hypothesise that this is driven by one, or a combination, of the following three effects. Firstly, our nineband photometric space is more resilient to spectroscopic selection biases than the four-band space considered in Hartley et al. (2020). Secondly, our deeper and more diverse spectroscopic compilation reduces the sensitivity of the recalibration procedure to strong (survey-specific) spectroscopic selection effects. Finally, the calibration method of Wright et al. (2020) is more resilient to spectroscopic selection effects than the method used in Hartley et al. (2020). We leave the exploration of these three possibilities to a future work.

A change in marginal $S_{8}$ constraints of $\left|\Delta S_{8}\right|=0.011$, with respect to the fiducial case, is nonetheless large in the context of our gold samples. As such, we extend this test further by implementing more stringent requirements on spectroscopic agreement. Our 'multispec3' gold sample consists only of photometric sources which are calibrated by spectra originating from at least three different spectroscopic surveys within our compilation. As stated in Sect. 2, this requirement places a strong restriction on spectroscopic agreement when coupled with our quality control requirement (Eq. (1)). For our multispec3 gold sample we find, again, a result that is consistent with our fiducial analysis: $S_{8}=0.721_{-0.045}^{+0.048}$, corresponding to $\left|\Delta S_{8}\right| \lesssim 0.005$, which is only slightly larger than the MCMC noise threshold. This result demonstrates the stability of our gold sample results and provides a strong indication that the marginal constraints on $S_{8}$ presented here are not biased by systematic effects nor catastrophic failures within the spectroscopic calibration sample.

While we have focussed our discussion here on the marginal $S_{8}$ constraints, we provide additional marginal and twodimensional posterior constraints for a subset of our parameter distributions in Appendix D. We explore other conclusions that can be drawn from our gold cosmological analyses, specifically around intrinsic alignments and the posterior probability distributions of $\Omega_{\mathrm{m}}$ and $\sigma_{8}$. Briefly, our gold sample marginal constraints show a reduced preference for low values of $\Omega_{\mathrm{m}}$, leading to a more consistent recovery of $\Omega_{\mathrm{m}} \approx 0.3$. In all of our gold analyses, the marginal constraints are good agreement $(|\Delta X|<$ $0.2 \sigma$ for all parameters $X)$, with the exception of the intrinsic alignment amplitude parameter $A_{\mathrm{IA}}$, which demonstrates up to $\left|\Delta A_{\mathrm{IA}}\right| \sim 1.0 \sigma$ differences among analyses. More importantly, however, our gold sample $A_{\mathrm{IA}}$ constraints are all consistent with $A_{\mathrm{IA}}=0$, unlike those from $\mathrm{H} 20$, who found $A_{\mathrm{IA}} \approx 1$. This updated constraint is in better agreement with recent work on intrinsic alignments (Fortuna et al. 2020), who predict an intrinsic alignment amplitude for KiDS of $0 \leq A_{\mathrm{IA}} \leq 0.2$. Finally, we find that differences in $S_{8}$ and $A_{\text {IA }}$ are correlated between our gold sample analyses and that stronger prior information on $A_{\text {IA }}$ would further improve the agreement between our respective gold sample analyses.

\section{Summary}

We present updated cosmological parameter constraints from the KiDS+VIKING-450 data set of Wright et al. (2019), estimated using updated redshift distributions following the methodology of Wright et al. (2020). For our fiducial analysis, we find a value of $S_{8}$ that is smaller but otherwise consistent with the value reported in the previous KiDS+VIKING-450 cosmological analysis of Hildebrandt et al. (2020): $S_{8}=0.716_{-0.038}^{+0.043}$ compared to $S_{8}=0.737_{-0.036}^{+0.040}\left(\left|\Delta S_{8}\right| \leq 0.6 \sigma\right)$. We note, however, that when the data and redshift distributions of Hildebrandt et al. (2020) are analysed using the updated redshift distribution bias parameters presented in Wright et al. (2020), their $S_{8}$ also shifts downward and is in better agreement with our fiducial analysis: $S_{8}=0.727_{-0.036}^{+0.039},\left|\Delta S_{8}\right| \leq 0.3 \sigma$. We explore the sensitivity of our results to systematic misrepresentation within the spectroscopic calibration data set by removing multiple spectroscopic subsamples (DEEP2, VVDS, zCOSMOS), each of which uniquely calibrate distinct portions of the colour-redshift space. We find that the results presented here are robust to pathological misrepresentation, whereby even the removal of DEEP2 is unable to 
cause a significant shift in $S_{8}:\left|\Delta S_{8}\right| \leq 0.2 \sigma$. We find that our results are consistent with the fiducial when performing the calibration using only certain ( $\mathrm{nQ}=4, \geq 99.5 \%$ confidence) spectroscopic redshifts and when performing an extremely conservative analysis that considers only photometric sources simultaneously calibrated by spectra from at least three different spectroscopic surveys: namely, $\left|\Delta S_{8}\right| \leq 0.011$ and $\left|\Delta S_{8}\right| \leq 0.005$, respectively. Overall, these results indicate that using the redshift calibration methodology of Wright et al. (2020), the pathological misrepresentation of photometric sources within the spectroscopic compilation is not able to produce significant changes in marginal constraints of $S_{8}$. Therefore, we find that it cannot reconcile the $\Delta S_{8} \approx 2.5 \sigma$ differences observed between cosmological parameters estimated using KiDS and Planck.

Acknowledgements. We thank the anonymous referee for their comments and suggestions, which have undoubtedly improved the quality of this manuscript. We acknowledge support from the European Research Council under grant numbers 770935 (AWH, HH, JvdB) and $647112(\mathrm{CH}) . \mathrm{HH}$ is also supported by a Heisenberg grant (Hi1495/5-1) of the Deutsche Forschungsgemeinschaft. $\mathrm{CH}$ also acknowledges support from the Max Planck Society and the Alexander von Humboldt Foundation in the framework of the Max PlanckHumboldt Research Award endowed by the Federal Ministry of Education and Research. KK acknowledges support from the Humboldt Foundation, and the hospitality of Imperial College London. This work is based on observations made with ESO Telescopes at the La Silla Paranal Observatory under programme IDs 100.A-0613, 102.A-0047, 179.A-2004, 177.A-3016, 177.A3017, 177.A-3018, 298.A-5015. The MICE simulations have been developed at the MareNostrum supercomputer (BSC-CNS) thanks to grants AECT-20062-0011 through AECT-2015-1-0013. Data products have been stored at the Port d'Informació Científica (PIC), and distributed through the CosmoHub webportal (cosmohub.pic.es).

\section{References}

Aihara, H., Arimoto, N., Armstrong, R., et al. 2018, PASJ, 70, S4

Alarcon, A., Sánchez, C., Bernstein, G. M., \& Gaztañaga, E. 2019, ArXiv e-prints [arXiv:1910.07127]
Audren, B., Lesgourgues, J., Benabed, K., \& Prunet, S. 2013, JCAP, 2, 001 Balestra, I., Mainieri, V., Popesso, P., et al. 2010, A\&A, 512, A12 Benítez, N. 2000, ApJ, 536, 571

Buchs, R., Davis, C., Gruen, D., et al. 2019, MNRAS, 489, 820

Edge, A., Sutherland, W., Kuijken, K., et al. 2013, The Messenger, 154, 32

Flaugher, B., Diehl, H. T., Honscheid, K., et al. 2015, AJ, 150, 150

Fortuna, M. C., Hoekstra, H., Joachimi, B., et al. 2020, MNRAS, submitted [arXiv:2003.02700]

Gruen, D., \& Brimioulle, F. 2017, MNRAS, 468, 769

Hartley, W. G., Chang, C., Samani, S., et al. 2020, MNRAS, 496, 4769

Hildebrandt, H., Erben, T., Kuijken, K., et al. 2012, MNRAS, 421, 2355

Hildebrandt, H., Viola, M., Heymans, C., et al. 2017, MNRAS, 465, 1454

Hildebrandt, H., Köhlinger, F., van den Busch, J. L., et al. 2020, A\&A, 633, A69

Hoyle, B., Gruen, D., Bernstein, G. M., et al. 2018, MNRAS, 478, 592

Jarvis, M., Bernstein, G., \& Jain, B. 2004, MNRAS, 352, 338

Kafle, P. R., Robotham, A. S. G., Driver, S. P., et al. 2018, MNRAS, 479, 3746

Kannawadi, A., Hoekstra, H., Miller, L., et al. 2019, A\&A, 624, A92

Kohonen, T. 1982, Biol. Cybern., 43, 59

Kuijken, K., Heymans, C., Dvornik, A., et al. 2019, A\&A, 625, A2

Le Fèvre, O., Cassata, P., Cucciati, O., et al. 2013, A\&A, 559, A14

Lilly, S. J., Le Brun, V., Maier, C., et al. 2009, ApJS, 184, 218

Lima, M., Cunha, C. E., Oyaizu, H., et al. 2008, MNRAS, 390, 118

McQuinn, M., \& White, M. 2013, MNRAS, 433, 2857

Miller, L., Kitching, T. D., Heymans, C., Heavens, A. F., \& van Waerbeke, L. 2007, MNRAS, 382, 315

Miller, L., Heymans, C., Kitching, T. D., et al. 2013, MNRAS, 429, 2858

Morrison, C. B., Hildebrandt, H., Schmidt, S. J., et al. 2017, MNRAS, 467, 3576

Newman, J. A. 2008, ApJ, 684, 88

Newman, J. A., Cooper, M. C., Davis, M., et al. 2013, ApJS, 208, 5

Planck Collaboration VI. 2020, A\&A, in press, https://doi.org/10.1051/ 0004-6361/201833910

Popesso, P., Dickinson, M., Nonino, M., et al. 2009, A\&A, 494, 443

Rau, M. M., Wilson, S., \& Mandelbaum, R. 2019, MNRAS, 491, 4768

Schneider, M., Knox, L., Zhan, H., \& Connolly, A. 2006, ApJ, 651, 14

Sánchez, C., \& Bernstein, G. M. 2018, MNRAS, 483, 2801

Tanaka, M., Coupon, J., Hsieh, B.-C., et al. 2018, PASJ, 70, S9

van den Busch, J. L., Hildebrandt, H., Wright, A. H., et al. 2020, A\&A, submitted [arXiv:2007.01846]

Vanzella, E., Cristiani, S., Dickinson, M., et al. 2008, A\&A, 478, 83

Wright, A. H., Hildebrandt, H., Kuijken, K., et al. 2019, A\&A, 632, A34

Wright, A. H., Hildebrandt, H., van den Busch, J. L., \& Heymans, C. 2020, A\&A, 637, A100 


\section{Appendix A: Cosmology and wrapper pipelines}

With this Letter, we release a new implementation of the KiDS cosmological analysis pipeline utilised by $\mathrm{H} 20$, which has been generalised for ease of use. The new pipeline, simply called CosmoPipe, is available online ${ }^{4}$. The package can be installed trivially with the provided master installation script and generates a clean working environment for each installation of the pipeline to avoid conflicts with, for example, existing python installations.

CosmoPipe contains the same analysis steps performed by H20. The pipeline utilises treecorr (Jarvis et al. 2004) for computation of cosmic shear correlation functions, and MontePython (Audren et al. 2013) for Markov-chain montecarlo (MCMC) analyses. For clarity, we outline the seven primary steps of the pipeline here.

1. Compute the 2D c-term for all survey patches and tomographic bins;

2. Compute the $1 \mathrm{D}$ c-term for all survey patches;

3. Compute $2 \mathrm{pt}$ shear correlation functions;

4. Construct the correlation function covariance matrix;

5. Prepare the data for input to MontePython MCMC: reformat the correlation functions, reformat the covariance matrix, prepare the montepython likelihood, reformat the Nz distributions, define the correlation function scale-cuts, and link any required treecorr files;

6. Run the MCMC;

7. Construct summary figures and statistics from the MCMC chains.

While this pipeline has been largely generalised, it is clear that some of these steps above are tailored for KiDS-like cosmological analyses. For example, the CosmoPipe is provided with a version of the public KiDS likelihood that has been preformatted to fit seamlessly into the CosmoPipe. The code will function equally well with an arbitrary likelihood, albeit with some additional preparation required on the user-side.

When performing an analysis such as (or indeed identical to) the one presented here, we also provide a wrapper package which links together the cosmological analysis pipeline package and the redshift calibration package of W20. This wrapper package ${ }^{5}$ contains one main script, Wright $2020 \mathrm{~b}$. sh, which performs the entirety of the analysis presented here. This script requires only that the user have the input photometric and spectroscopic calibration data sets supplied. It performs (with one command) the full gambit of analyses required for this study. These steps include:

1. redshift calibration;

2. gold sample selection;

3. installation of CosmoPipe;

4. preparation of CosmoPipe for the different gold sample runs;

5. running CosmoPipe;

6. outputting of figures present in this Letter.

Some additional input parameters to the CosmoPipe are also encoded in the wrapper package, such as the various redshift distribution and multiplicative shear bias priors given discussed in Appendix B.

\section{Appendix B: Gold sample priors}

\section{B.1. Mean redshift distribution biases}

Redshift distribution bias is a significant uncertainty in cosmic shear analyses, as coherent bias in redshift distribution means is directly reflected as a bias in $S_{8}$. In H20, redshift distributions were assigned a zero-mean (' $\delta z=0$ ') Gaussian prior, whose width (per tomographic bin) was estimated via a spatial bootstrap method (see Sect. 3.2 of H20). These priors are applicable to the direct calibration implemented in their work, but need not be reflective of the biases in our SOM-based redshift calibration procedure.

Fortunately, W20 present estimates of the redshift calibration biases for our fiducial gold sample. In brief, these redshift distribution bias estimates are made using simulations of the KiDS photometric and spectroscopic compilations from van den Busch et al. (2020). These simulations generate 100 independent realisations of the three main spectroscopic linesof-sight within KiDS, which are then used to calibrate a mock KiDS photometric sample. At all steps in this process, samples are constructed to match the real KiDS photometric and spectroscopic data in all possible manners: magnitude, colour, selection effects, weights, redshift distributions, etc. This simulation therefore allows the estimation of mean redshift distribution biases, include uncertainties and biases introduced by photometric noise, sample variance, spectroscopic selection effects, spectroscopic incompleteness, and Poisson sampling. Tests performed in W20 suggest that the dominant sources of calibration uncertainty are photometric noise and spectroscopic selection effects. Using the recovered mean redshift distribution bias estimates (and uncertainties), we are able to construct new, more informative, non-zero-mean ( $' \delta z \neq 0$ ') redshift distribution bias priors for our cosmic shear analysis. These priors are presented in Table B.1.

As each of the zCOSMOS, VVDS, and DEEP2 samples were simulated by van den Busch et al. (2020), we are able to also calibrate the redshift bias priors for the three gold samples which exclude these subsamples: our 'nozCOSMOS', 'noVVDS', and 'noDEEP2' gold samples. However, the simulations of van den Busch et al. (2020) did not include estimates of redshift quality, so we are unable to construct a more informative prior for the 'speczquality4' gold sample. Similarly, as they do not simulate every spectroscopic subsample within KiDS, construction of a new 'multispec3' prior is also not possible. In each of these cases, we are therefore required to default back to the ' $\delta z=0$ ' priors of $\mathrm{H} 20$.

To be conservative, we opt to double the uncertainties on the bias found in the simulations when constructing our priors. We opt to utilise these new priors, where possible, as they represent our current best-estimate of the true redshift bias parameters inherent to the recalibration method and samples used here, despite the limitations of the simulations used (van den Busch et al. 2020; Wright et al. 2020). We note, however, that the biases are typically small, being on the order of $\delta z \lesssim 0.01$ for the majority of samples and bins, meaning that the $\mathrm{H} 20 \delta z=0$ prior is nonetheless a reasonable approximation (and therefore unlikely to be a source of bias in the cases where we are required to use that prior; i.e. speczquality 4 and multispec3).

\section{B.2. Multiplicative shear bias}

As each of our gold selections produces a different subset of the full photometric sample, this requires a new computation

\footnotetext{
4 https://www.github.com/AngusWright/CosmoPipe

5 https://www.github.com/AngusWright/CosmoWrapper
} 
Table B.1. Updated redshift distribution bias priors parameters used in different gold sample analyses.

\begin{tabular}{lcrrrrr}
\hline \hline \multirow{2}{*}{$\begin{array}{c}\text { Gold } \\
\text { sample }\end{array}$} & \multicolumn{5}{c}{ Tomographic redshift bias prior $\delta z$} \\
\cline { 3 - 7 } & & \multicolumn{1}{c}{ Bin1 } & \multicolumn{1}{c}{ Bin2 } & \multicolumn{1}{c}{ Bin3 } & \multicolumn{1}{c}{ Bin4 } & \multicolumn{1}{c}{ Bin5 } \\
& Fiducial & $0.000 \pm 0.010$ & $0.002 \pm 0.012$ & $0.013 \pm 0.012$ & $0.011 \pm 0.008$ & $-0.006 \pm 0.010$ \\
\multirow{4}{*}{$\delta z \neq 0$} & KV450-DIR & $0.047 \pm 0.010$ & $0.025 \pm 0.008$ & $0.032 \pm 0.010$ & $-0.004 \pm 0.008$ & $-0.013 \pm 0.008$ \\
& NoDEEP2 & $-0.001 \pm 0.010$ & $0.002 \pm 0.012$ & $-0.002 \pm 0.012$ & $-0.009 \pm 0.010$ & $-0.015 \pm 0.010$ \\
& noVVDS & $0.001 \pm 0.010$ & $0.001 \pm 0.012$ & $0.024 \pm 0.014$ & $0.014 \pm 0.010$ & $-0.007 \pm 0.012$ \\
& nozCOSMOS & $0.005 \pm 0.026$ & $0.005 \pm 0.016$ & $0.032 \pm 0.014$ & $0.030 \pm 0.010$ & $0.002 \pm 0.012$ \\
\hline \multicolumn{2}{c}{ All $\delta z=0$} & $0.000 \pm 0.039$ & $0.000 \pm 0.023$ & $0.000 \pm 0.026$ & $0.000 \pm 0.012$ & $0.000 \pm 0.011$ \\
\hline
\end{tabular}

Notes. Priors are Gaussian $(\mu \pm \sigma)$. Parameters are determined from the simulations of van den Busch et al. (2020) as described in W20, except that we double the simulation bias standard deviations when constructing our priors. For samples where we want to replicate previous analyses, we implement the prior from $\mathrm{H} 20$ ('All $\delta z=0$ ').

Table B.2. Multiplicative shear bias parameters used for each of our gold sample analyses.

\begin{tabular}{lcrrrr}
\hline \hline \multirow{2}{*}{$\begin{array}{l}\text { Gold } \\
\text { sample }\end{array}$} & \multicolumn{5}{c}{ Multiplicative shear bias parameter } \\
\cline { 2 - 6 } Fiducial & $-0.0145 \pm 0.0200$ & $-0.0176 \pm 0.0200$ & $-0.0125 \pm 0.0200$ & $0.0045 \pm 0.0200$ & $0.0122 \pm 0.0200$ \\
NoDEEP2 & $-0.0137 \pm 0.0200$ & $-0.0162 \pm 0.0200$ & $-0.0112 \pm 0.0200$ & $0.0054 \pm 0.0200$ & $0.0130 \pm 0.0200$ \\
noVVDS & $-0.0143 \pm 0.0200$ & $-0.0172 \pm 0.0200$ & $-0.0116 \pm 0.0200$ & $0.0047 \pm 0.0200$ & $0.0125 \pm 0.0200$ \\
nozCOSMOS & $-0.0143 \pm 0.0200$ & $-0.0159 \pm 0.0200$ & $-0.0106 \pm 0.0200$ & $0.0053 \pm 0.0200$ & $0.0135 \pm 0.0200$ \\
speczquality4 & $-0.0141 \pm 0.0200$ & $-0.0163 \pm 0.0200$ & $-0.0121 \pm 0.0200$ & $0.0043 \pm 0.0200$ & $0.0125 \pm 0.0200$ \\
multispec3 & $-0.0158 \pm 0.0200$ & $-0.0203 \pm 0.0200$ & $-0.0173 \pm 0.0200$ & $-0.0033 \pm 0.0200$ & $-0.0012 \pm 0.0200$ \\
\hline
\end{tabular}

of the multiplicative and additive shear biases, shear correlation functions, covariances, etc. Each of these is incorporated into the pipeline processing, with the exception of the multiplicative shear bias estimation. In H20, multiplicative shear biases are computed using the methodology and simulations of Kannawadi et al. (2019). We invoke the same procedure, thereby generating a bespoke set of multiplicative shear-bias parameters for each of our gold selections, albeit outside of our wrapper pipeline. These bias parameters are given in Table B. 2 for each of our gold samples. We note that in all cases, we have chosen to implement the same $m$-bias uncertainty as used in H20: $\Delta m=0.02$ for all tomographic bins.

We recognise that this implementation of the $m$-bias estimation may be sub-optimal: the gold selections are strongly colourdependent and our current simulation-set for estimating multiplicative bias is entirely mono-chromatic (using only the $r$-band imaging and fluxes). However these simulations nonetheless represent the state-of-the-art within KV450 and we leave exploration of how the $m$-biases change with multi-colour simulations for future studies. These $m$-bias parameters are required as input to our cosmology pipeline, and so are documented here. Overall, the gold sample multiplicative biases are very similar, with only the multispec 3 calibration differing from the fiducial by more than $\Delta m \sim 0.002$. In all cases, the different $m$-bias values are well within the assumed multiplicative bias uncertainty used here.

\section{Appendix C: Gold sample representation statistics}

In this work, we test the sensitivity of our cosmological parameter estimates to differently constructed gold samples within KV450. Each of these gold samples produces a subset of the available photometric data and results in a different set of tomographic redshift distributions. We present these representation statistics and corresponding redshift distributions means here in Table C. 1 .

The combinations of mean redshift and representation statistics tell an interesting story about which photometric data are removed by each of our gold sample definitions. There is a clear correlation between the removal of photometric data and a subsequent decrease in the mean redshift of the tomographic bins. The most obvious examples of this are in the cases of our noDEEP2 and multispec 3 samples, where the gold selection removes $30 \%$ and $45 \%$ of the fiducial $n_{\text {eff }}$ in the fifth tomographic bin, respectively. These samples also show the largest redshift distribution shifts within our gold samples: $\Delta\langle z\rangle \sim 0.05$ in the fifth tomographic bin. The suggests that the gold-sample definition is preferentially removing truly high-redshift sources from the photometric sample, as expected. This is indicative of the robustness of the joint redshift distribution estimation and gold selection; unlike the case of the redshift calibration in $\mathrm{H} 20$, each combination of gold-sample and redshift distribution presented here is compatible, and differences in sample mean redshifts are not indications of bias in the redshift calibration methodology. This is an important distinction between the different redshift distributions presented here and in $\mathrm{H} 20$.

Finally, we note the impact that the reduced effective number density of each gold sample has on our posterior constraint of $S_{8}$. The multispec 3 subsample, for example, has roughly $50 \%$ of the photometric $n_{\text {eff }}$ of the KV450-DIR sample per tomographic bin, but shows only a $\sim 35 \%$ larger uncertainty on $S_{8}$. This is in agreement with the results of H20, who found that the KV450-DIR $S_{8}$ uncertainty was limited in equal measure by both statistical and systematic uncertainties. 
Table C.1. Mean tomographic redshifts and representation statistics of photometric source galaxies within each of our gold samples.

\begin{tabular}{|c|c|c|c|c|c|c|c|c|c|c|}
\hline \multirow{2}{*}{$\begin{array}{l}\text { Gold } \\
\text { sample }\end{array}$} & \multicolumn{5}{|c|}{$n_{\mathrm{eff}}^{\mathrm{gold}} / n_{\mathrm{eff}}^{\mathrm{ref}}(\%)$} & \multicolumn{5}{|c|}{$\langle z\rangle$} \\
\hline & Bin1 & $\operatorname{Bin} 2$ & Bin3 & Bin 4 & Bin5 & Bin1 & Bin2 & Bin3 & Bin 4 & Bin5 \\
\hline KV450-DIR & 100.0 & 100.0 & 100.0 & 100.0 & 100.0 & 0.369 & 0.463 & 0.643 & 0.806 & 0.973 \\
\hline Fiducial & 78.6 & 82.1 & 79.2 & 82.3 & 91.6 & 0.236 & 0.379 & 0.537 & 0.766 & 0.948 \\
\hline nozCOSMOS & 93.4 & 92.2 & 92.0 & 88.3 & 91.5 & 0.214 & 0.371 & 0.529 & 0.755 & 0.945 \\
\hline noDEEP2 & 97.7 & 96.2 & 88.6 & 79.5 & 72.7 & 0.237 & 0.374 & 0.516 & 0.737 & 0.908 \\
\hline noVVDS & 97.1 & 92.4 & 86.2 & 88.1 & 91.4 & 0.237 & 0.373 & 0.537 & 0.766 & 0.951 \\
\hline speczquality4 & 95.0 & 92.0 & 87.2 & 86.8 & 89.4 & 0.231 & 0.367 & 0.524 & 0.756 & 0.941 \\
\hline multispec3 & 71.2 & 72.7 & 65.0 & 55.4 & 54.5 & 0.226 & 0.369 & 0.515 & 0.737 & 0.906 \\
\hline
\end{tabular}

Notes. Representation is defined using the effective number density of sources for cosmic shear studies, $n_{\text {eff }}$, in each of the gold samples relative to a reference sample $n_{\text {eff. }}$. For the fiducial representation statistic, we use the full KV450 photometric data set for reference (i.e. $n_{\text {eff }}^{\text {fid }} / n_{\text {eff }}^{\text {all }}$ ), while all other gold sample representations use the fiducial for reference (i.e. $n_{\text {eff }}^{\text {gold }} / n_{\text {eff }}^{\text {fid }}$ ). The statistics are all given per tomographic bin. The table demonstrates that each of our nozCOSMOS, noVVDS, and noDEEP2 gold samples has preferentially removed a different section of the colourspace. This is joined, however, by a shift in the mean redshift of the tomographic bin, indicating that the loss of the colour redshift space has been accounted for in the reconstruction. As expected, the multispec 3 selection is highly restrictive, removing $30-45 \%$ of the fiducial photometric $n_{\text {eff }}$ in every bin.

\section{Appendix D: Additional marginal constraints}

Here we present a subset of the posterior constraints from a subset of our gold sample analyses. In Figs. D.1 and D.2, we show 4 of the 14 cosmological and nuisance parameters which are used by our likelihood model $\left(A_{\mathrm{IA}}, n_{\mathrm{s}}, h\right.$, and $\left.\ln 10^{10} A_{\mathrm{s}}\right)$, as well as 3 derived parameters $\left(\Omega_{\mathrm{m}}, \sigma_{8}\right.$, and $\left.S_{8}\right)$, in their marginal and $2 \mathrm{D}$ projections respectively. The marginal mean and standard deviations of these posterior distributions are also provided in Table D.1. For an in-depth description of the likelihood used here, see H20. We have selected these parameters to show because they are of cosmological interest or they are not priordominated in our analysis. In particular, we opt to not show the various redshift distribution-bias posteriors, as they follow their priors exactly in all our gold sample analyses. In this way, our results reinforce those of previous works: cosmic shear correlation functions cannot self-calibrate redshift distribution biases.

The marginal distributions from each of our gold samples in Fig. D.1 are in good agreement. Comparing the various gold sample analyses to our two 'KV450-DIR' runs, which use data vectors and redshift distributions equivalent to those in $\mathrm{H} 20$, we see some interesting differences. Firstly, we note that the gold samples no longer demonstrate a preference for small values of the matter density parameter, $\Omega_{\mathrm{m}} \sim 0.18$. Instead, our gold marginal distributions all peak at values $\Omega_{\mathrm{m}} \sim 0.3$, in much better agreement with concordance cosmological parameters. This has a subsequent effect on the marginal distribution of $\sigma_{8}$, causing it to be considerably narrower for our gold analysis than in the KV450-DIR cases; we find $\sigma_{8}=0.762_{-0.180}^{+0.070}$ compared to $\sigma_{8}=0.836_{-0.218}^{+0.132}$.

Focussing on the marginal constraints on $A_{\mathrm{IA}}$, we see that this parameter shows the greatest variation within our gold sample analyses. Interestingly, however, we note that only the results of 'KV450-DIR' (i.e. KV450-DIR using the fiducial redshift bias priors) demonstrate a preference for non-zero values of $A_{\mathrm{IA}}$. In all other cases, the marginal constraints are consistent with $A_{\mathrm{IA}}=$ 0 ; in agreement with recent work on intrinsic alignments within KiDS (Fortuna et al. 2020), who advocate $0 \leq A_{\text {IA }} \leq 0.2$.

Furthermore, we note a correlation between the values of $A_{\text {IA }}$ and $S_{8}$ in the marginal distributions. Looking to the 2D posteriors in Fig. D.2, the degeneracy between these two parameters is clearer. Interestingly, the $A_{\mathrm{IA}}$ versus $S_{8}$ joint posterior (for the 'KV450-DIR', 'SOM-Gold Fiducial', and 'SOM-Gold noDEEP2' samples shown) demonstrates that a stronger prior on the value of $A_{\text {IA }}$ would likely bring the value of $S_{8}$ in these analyses into even closer agreement. This, in turn, suggests that the freedom of our intrinsic alignment parameter does not improve the agreement of $S_{8}$ between our different gold samples; rather, it is the opposite effect. For the purposes of demonstration only, we present in Table D.2 the marginal posterior constraints on $S_{8}$ for posterior samples within $0 \leq A_{\mathrm{IA}} \leq 0.2$, compared to the full posterior results (which invokes an intrinsic alignment amplitude prior of $\left.-6 \leq A_{\mathrm{IA}} \leq 6\right)$. This clearly demonstrates the interplay between $A_{\mathrm{IA}}$ and $S_{8}$; for the restricted prior, we find a

Table D.1. Marginal parameter means and standard deviations for the subset of parameters shown in Fig. D.1.

\begin{tabular}{lcrrrrr}
\hline \hline Parameter & KV450-DIR & Fiducial & $\begin{array}{c}\text { nozCOSMOS } \\
\delta z \neq 0\end{array}$ & noVVDS & noDEEP2 & $\begin{array}{c}\text { KV450-DIR } \\
\delta z=0\end{array}$ \\
\hline$A_{\mathrm{IA}}$ & $0.282 \pm 0.594$ & $-0.344 \pm 0.695$ & $-0.366 \pm 0.650$ & $0.198 \pm 0.665$ & $-0.627 \pm 0.775$ & $0.959 \pm 0.671$ \\
$n_{\mathrm{s}}$ & $1.044 \pm 0.130$ & $1.023 \pm 0.133$ & $1.042 \pm 0.136$ & $1.020 \pm 0.137$ & $1.072 \pm 0.130$ & $1.032 \pm 0.131$ \\
$h$ & $0.747 \pm 0.049$ & $0.742 \pm 0.049$ & $0.743 \pm 0.049$ & $0.741 \pm 0.050$ & $0.741 \pm 0.049$ & $0.748 \pm 0.048$ \\
$\ln 10^{10} A_{\mathrm{S}}$ & $3.158 \pm 0.864$ & $2.816 \pm 0.806$ & $2.762 \pm 0.795$ & $2.617 \pm 0.715$ & $2.653 \pm 0.743$ & $3.099 \pm 0.882$ \\
$\Omega_{\mathrm{m}}$ & $0.249 \pm 0.082$ & $0.282 \pm 0.085$ & $0.286 \pm 0.085$ & $0.305 \pm 0.085$ & $0.291 \pm 0.081$ & $0.259 \pm 0.087$ \\
$\sigma_{8}$ & $0.834 \pm 0.156$ & $0.768 \pm 0.143$ & $0.765 \pm 0.145$ & $0.739 \pm 0.132$ & $0.743 \pm 0.134$ & $0.833 \pm 0.160$ \\
$S_{8}$ & $0.728 \pm 0.035$ & $0.716 \pm 0.038$ & $0.718 \pm 0.041$ & $0.719 \pm 0.038$ & $0.707 \pm 0.042$ & $0.739 \pm 0.036$ \\
\hline
\end{tabular}



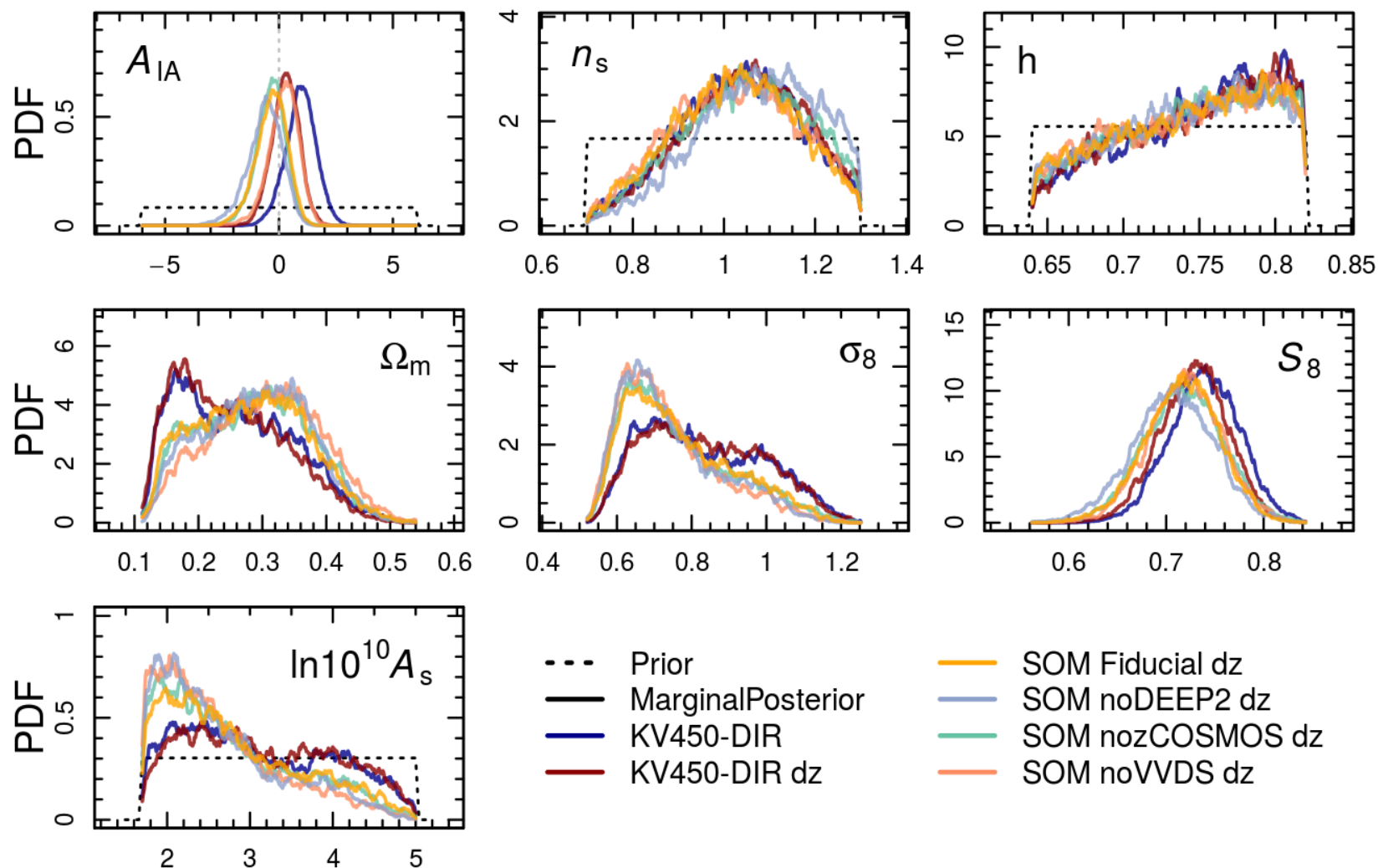

SOM Fiducial $\mathrm{dz}$ SOM noDEEP2 dz SOM nozCOSMOS dz SOM noVVDS dz

Fig. D.1. Marginal posterior distributions for a subset of the cosmological, nuisance, and derived parameters used in our cosmological model. Coloured lines represent the marginal distributions from various samples. Dashed lines show the priors for all non-derived parameters. There is a clear difference between the marginal distributions of the gold and full-sample ('KV450-DIR') analyses. We note in particular that the previously observed preference within KV450 for small values of the matter density parameter $\Omega_{\mathrm{m}}$ is removed in our gold analyses. The gold analyses also prefer a lower value of $A_{\mathrm{IA}}$, consistent with 0 in all cases.

maximal deviation between marginal constraints of $\left|\Delta S_{8}\right| \lesssim$ 0.005 , only marginally above the MCMC noise. Nonetheless, we caution against the over-interpretation of these constraints, as a stronger prior on $A_{\mathrm{IA}}$ is not yet justifiable. Analyses such as that of Fortuna et al. (2020) invoke a number of assumptions that prohibit the use of their constraints as prior distributions.
Table D.2. For the purposes of demonstration only, the marginal constraints of $S_{8}$ with our fiducial and reduced $A_{\text {IA }}$ prior.

\begin{tabular}{cccc}
\hline \hline \multirow{2}{*}{ Gold } & \multicolumn{2}{c}{ Marginal $S_{8}$ constraint } \\
\cline { 2 - 4 } & sample & $-6 \leq A_{\mathrm{IA}} \leq 6$ & $0 \leq A_{\mathrm{IA}} \leq 0.2$ \\
\hline \multirow{3}{*}{$\delta z \neq 0$} & KV450-DIR & $0.728 \pm 0.035$ & $0.729 \pm 0.035$ \\
& Fiducial & $0.716 \pm 0.038$ & $0.724 \pm 0.036$ \\
& nozCOSMOS & $0.718 \pm 0.041$ & $0.728 \pm 0.038$ \\
& noVVDS & $0.719 \pm 0.038$ & $0.722 \pm 0.037$ \\
& noDEEP2 & $0.707 \pm 0.042$ & $0.724 \pm 0.038$ \\
\hline \multirow{3}{*}{$\delta z=0$} & KV450-DIR & $0.739 \pm 0.036$ & $0.730 \pm 0.034$ \\
& Fiducial & $0.714 \pm 0.039$ & $0.722 \pm 0.036$ \\
& speczquality4 & $0.727 \pm 0.039$ & $0.729 \pm 0.037$ \\
& multispec3 & $0.721 \pm 0.044$ & $0.725 \pm 0.041$ \\
\hline
\end{tabular}

Notes. This shows that at fixed values of $A_{\mathrm{IA}}$, each of the gold samples are in agreement with respect to $S_{8}$ and, therefore, that the differences seen in $S_{8}$ are not improved by the freedom of $A_{\mathrm{IA}}$ in our cosmological model. 


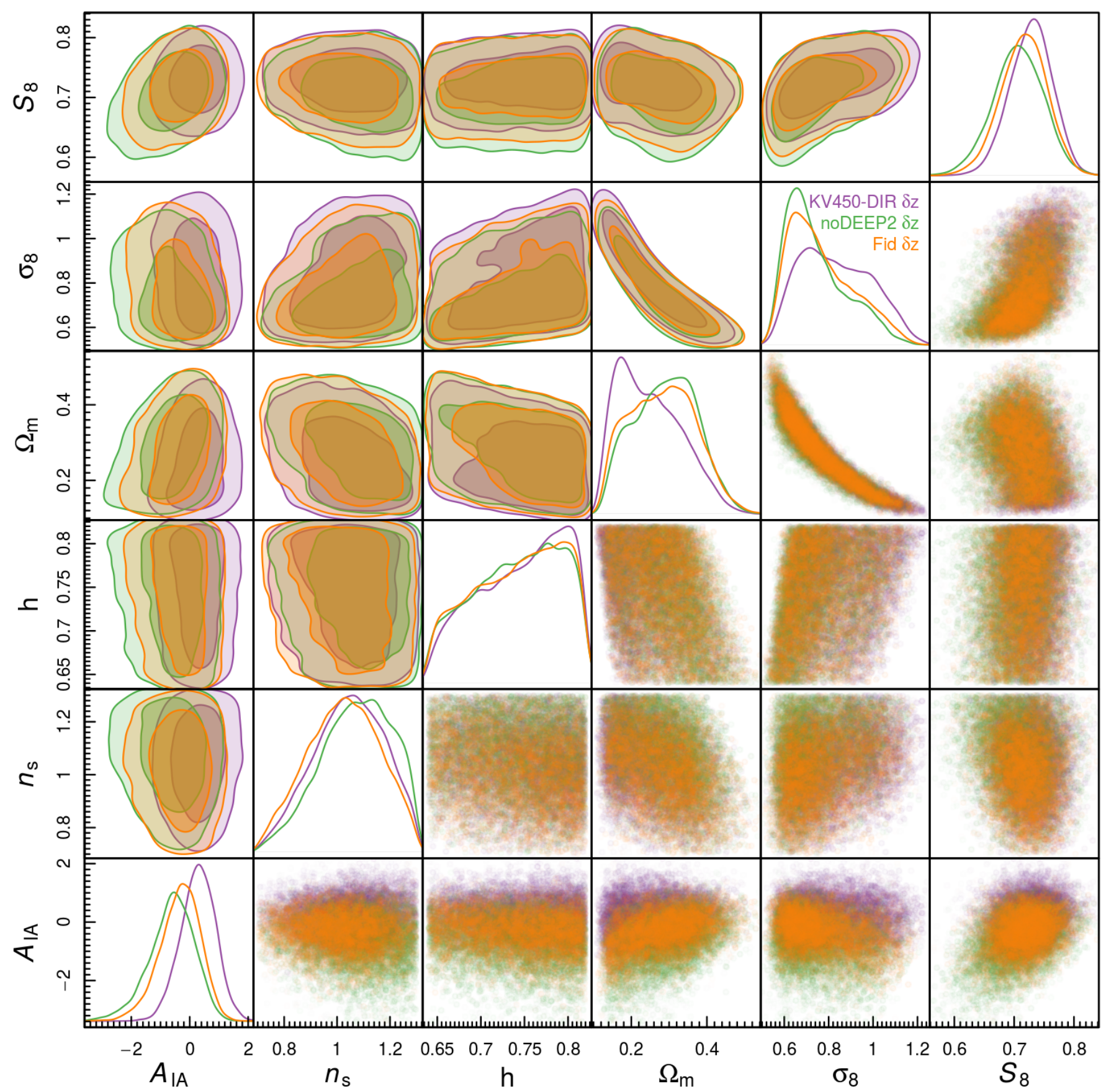

Fig. D.2. Marginal and 2D posterior distributions for a subset of the cosmological, nuisance, and derived parameters used in our cosmological model. We restrict this figure to only the 'KV450-DIR $\delta z$ ' (purple), 'SOM-Gold Fidicial $\delta z$ ' (gold), and 'SOM-Gold noDEEP2 $\delta z$ ' (green) analyses, as these three analyses encompass the range of posteriors in all of our gold sample analyses (and show similar degeneracies). The joint posterior of $A_{\mathrm{IA}}$ and $S_{8}$ demonstrates that, were a stronger prior on $A_{\mathrm{IA}}$ justifiable, agreement between the various gold samples would be even higher. 